\title{
EFECTO DE UN PROGRAMA DE EJERCICIOS CON PESAS EN EL PORCENTAJE DE GRASA Y LA FUERZA EN MUJERES MAYORES
}

\author{
Magally Márquez Barquero y Cinthya Campos Salazar \\ Escuela de Educación Física y Deportes \\ Universidad de Costa Rica, San José, Costa Rica \\ E-mailmmarquez@cariari.ucr.ac.cr
}

\begin{abstract}
Resumen
Márquez, M. y Campos, C. (2001). Efecto de un programa de ejercicios con pesas en el porcentaje de grasa y la fuerza en mujeres entre los 50 y 59 años. Revista de Ciencias del Ejercicio y la Salud, 1(2), 31-37. El propósito del estudio fue determinar los efectos crónicos del entrenamiento con pesas sobre el porcentaje de grasa y la fuerza. Los participantes, de sexo femenino, entrenadas ( $n=10$, edad $54.6 \pm 4.19$ años, un peso inicial promedio de $66.08 \pm 12.50 \mathrm{Kg}$ y una talla promedio de $1.585 \pm 0.0754 \mathrm{~m}$.), fueron divididos aleatoriamente en dos grupos: $50 \%$ de $5 R M(n=5), 80 \%$ de $5 R M(n=5)$ y un pre test de registro de línea base. Las variables medidas fueron fuerza y porcentaje de grasa. Ambos grupos realizaron dos sesiones por semana durante doce semanas. Los ejercicios para ambos grupos fueron press de banca horizontal, extensión de rodilla, flexión de rodilla y jalón de polea frontal. El volumen de ejercicio total por sesión fue de 2550 unidades para el grupo que trabajo al $50 \%$ (3 series $x 17$ rep. $X$ 50\%) y 2560 para el que trabajo al 80\% (4 series x 8 repeticiones $x$ 80\%). La fuerza se midió con el Test de 5 RM y el porcentaje de grasa fue estimado con la técnica de toma de siete pliegues cutáneos con el calibrador manual de piel marca Lange y subsecuentemente estimado usando la ecuación Leger y Cloutier para mujeres mayores de 50 años. Resultados: Para las pruebas de fuerza, press de banca, flexión de rodilla y extensión de rodilla hubo mejora significativa, tanto para el grupo que se ejercitó al $50 \%$ como al $80 \%$ de intensidad ( $p<0.05$ ). Mientras que para la prueba halón de polea al frente el cambio entre mediciones $(p<0.05)$, siendo mayor para el grupo que se ejercitó al $80 \%$ de intensidad. En cuanto al porcentaje de grasa, disminuyó ( $p<0.05)$ similarmente a ambas intensidades de ejercicio. En conclusión, a intensidades del 50\% como del $80 \%$ de 5RM, disminuye el porcentaje de grasa y aumenta la fuerza, en mujeres con una edad comprendida entre los 50 y 59 anos quienes realizaron una misma carga total de ejercicio con pesas. Los hallazgos sugieren que el entrenamiento con pesas a cargas altas, beneficia a los grandes grupos musculares. PALABRAS CLAVES: Fuerza, porcentaje de grasa, volumen total de ejercicio, 5RM.
\end{abstract}

\section{INTRODUCCIÓN}

El beneficio del entrenamiento con pesas en la población mayor ha sido ampliamente estudiado. Inicialmente la tendencia de los estudios fue determinar el beneficio del entrenamiento con pesas en el fortalecimiento muscular (Peterson, 1991; Smith y otros, 1991; Heislein, 1994; Meléndez,2000, Booth y otros,2001). Una vez comprobada la ganancia de fuerza que la población mayor experimenta debido a la práctica de ejercicio con pesas, de hasta un $110 \%$ de ganancia, con sujetos entre 60 y 72 años de edad con 12 semanas de entrenamiento (Meredith y otros,
1989;Fiatarone y otros, 1990), se realizan estudios que intentan determinar si tales programas provocan beneficios en otras variables. Como por ejemplo el aumento de la densidad ósea (Layne y Nelson, 1999) logrando mantener los niveles de calcio y fósforo normales en el cuerpo, si se logra mejorar la capacidad funcional física o facilitar el cumplimiento de las actividades básicas diarias (Astrand, 1992), beneficios de a nivel cardiovascular como su efecto en el retorno venoso (Gutiérrez, 1999), sobre la presión arterial y frecuencia cardiaca (Campos y otros, 1999), sobre el perfil de lípidos (Campos y otros, 1998), a nivel afectivo, como por ejemplo 
la autoestima (Nichols y otros, 1995) así como también en variables cognitivas al realizar programas de ejercicio con pesas (Morel, 1999).

En relación con el entrenamiento con pesas, la composición corporal y la población mayor, la literatura es escasa. Cabe citar que conforme avanza la edad se presenta un incremento en el peso corporal, del cual, la mayor parte es de tipo graso. $\mathrm{Y}$ se asocia además con disminución de la masa libre de grasa (Wilmore \& Costill,1999).

Conforme la mujer envejece, es más propensa al sobrepeso y a depositar grasa en los adipositos en el abdomen especialmente. Se estima que la masa magra declina del $10 \%$ al $20 \%$ con el envejecimiento y durante este periodo se presenta una acumulación del tejido adiposo, lo cual a su vez se acompaña con una disminución en el nivel de actividad física diario.

Westcott (1991), por su parte, realizó un estudio con 72 mujeres con sobrepeso, que consistió en 8 semanas de actividad física, un grupo de las participantes realizó 30 minutos de ejercicio aeróbico y otro grupo realizó un programa combinado de 15 minutos de ejercicio aeróbico y 15 minutos de ejercicio con pesas. El grupo que practicó ejercicio aeróbico perdió 3.5 libras, de las cuales 3 libras fueron de tejido graso y 0.5 libras de fue masa muscular; el grupo que practicó ejercicio combinado perdió 8 libras de peso, aun cuando, en los datos finales presentaron en promedio un aumento de 2 libras de masa muscular.

Posteriormente, Astrup (1999), realizó un estudio descriptivo con mujeres posmenopáusicas y encontró que las mujeres con niveles altos de actividad física presentan menores porcentajes de grasa corporal.

Hunter y otros (2000) por su parte, realizaron un estudio en el cual encontraron que el entrenamiento con pesas, durante 16 semanas, provocó en mujeres mayores de 50 años aumentar la masa libre de grasa e incrementar la oxidación de lípidos. Los autores (2000) aseguran que es probable que el peso corporal no cambie, pero que sí decrece el porcentaje de grasa corporal. Sin embargo los autores no reportan detalles del manejo de las cargas del programa de ejercicio.

El interés de estudiar cómo influye el ejercicio de fuerza en otras variables, como las citadas, parte de la adaptabilidad del sistema músculo esquelético al entrenamiento de fuerza que ha mostrado la población mayor (Faulkner \& White, 1990; Israel,1992; Wilmore \& Costill, 1999) y los beneficios asociados al fortalecimiento muscular, como son: el incremento en el grado de independencia, reducción de la incidencia a caídas, contrarrestar la debilidad muscular logrando inhibir la sensación de dolor muscular y articular, disminuir el umbral de fatiga, reduciendo el riesgo a lesiones por sobreesfuerzo y cansancio.

Por mucho tiempo se manejó el concepto que la ganancia de fuerza se lograba únicamente al trabajar con cargas superiores o iguales al 70\% de intensidad, mas en 1999 se publica un estudio realizado con población mayor en el cual se determinó ganancia de fuerza muscular tanto en aquellos participantes que se ejercitaron a baja intensidad, $30 \%$ de $5 \mathrm{RM}$, como a alta intensidad, $70 \%$ de $5 \mathrm{RM}$ (Kaver y otros,1999). La ganancia de fuerza fue similar en ambos grupos, otros estudios han encontrado resultados similares (Laidlaw y otros, 1999; Bemben y otros, 2000; Russo y otros, 2001).

La razón de tales resultados, según los estudios citados, es debido al control del volumen total de trabajo por sesión, es decir, el grupo que se ejercita a baja intensidad realiza en total la misma cantidad de trabajo que el grupo que se ejercita a alta intensidad.

Sin embargo, los estudios anteriores no reportan el intervalo de tiempo en las series de ejercicio, variable que será controlada en el presente estudio.

La propuesta del estudio es aplicar un programa de ejercicio con pesas a diferentes intensidades, con el mismo volumen de trabajo por sesión y sin descanso entre las series de ejercicio, alternando grupos musculares.

El objetivo de aplicar dos intensidades de entrenamiento distintas, controlando el volumen de trabajo por sesión y sin descanso en las series de ejercicio, es considerando que 
los resultados en la variable porcentaje de grasa corporal sean similares a los encontrados en la variable fuerza muscular (Kaver y otros, 1999; Bemben, 2000; Laidlaw y otros, 1999; Russo y otros, 2001); lo cual resultaría de gran beneficio para la población en estudio, pues se tendría la posibilidad de que tanto a intensidades bajas como altas logre beneficiarse tanto en el fortalecimiento muscular como la reducción del porcentaje de grasa subcutánea.

\section{METODOLOGÍA}

\section{Sujetos}

Participaron en el estudio 10 mujeres que asistían al programa de acondicionamiento con pesas de la Universidad de Costa Rica, y tenían ocho meses de participar en el programa. La edad promedio y desviación estándar de las participantes fue de $54.6 \pm 4.2$ años.

\section{Instrumentos de medición}

Calibrador manual marca Lange con precisión constante de $10 \mathrm{gr} \times \mathrm{mm}^{2} \pm 1 \mathrm{~mm}$. Para la interpretaciones de la mediciones se utilizó la ecuación de Leger y Cloutier para mujeres mayores de 50 años. La ecuación es la siguiente: $\%$ grasa $=100 \times(4.95 /(1.1339-$ $0.0645 \times \log y)-4.5)$, donde " $y$ ” es la suma de pliegues. Para la medición del peso se utilizó una báscula electrónica marca $\mathrm{A} \& \mathrm{D}$, modelo UC 300 con precisión $0.1 \mathrm{lb}$.

Prueba submáxima de 5 repeticiones máximas (5RM) para los cinco ejercicios que conformaron el programa: extensión de rodilla, flexión de rodilla, press de banca horizontal y halón de polea frontal. La utilización de una prueba submáxima responde a la recomendación del ACSM (2000) en relación con los riesgos de la aplicación de pruebas máximas desde el punto de vista cardiovascular, a saber, isquemia cardiaca, accidente vascular periférico, arritmias, trombosis coronaria, siendo los accidentes cardiovascular la principal causa de morbimortalidad en países desarrollados $y$ en desarrollo.

\section{Procedimientos de medición}

Para el reclutamiento de la muestra de participantes se explicaron las características del estudio a las participantes del programa y aquellas personas interesadas en participar dieron su consentimiento formal. Cada participante fue medida en las cinco variables mencionadas, 2 veces antes de iniciar el programa de ejercicios. Se realizó una medición 2 semanas antes de iniciar el programa "pretest 1 " y se realizó otra medición 1 semana antes de iniciar con el programa "pretest 2". Seguidamente se aleatorizaron las participantes a los tratamientos. Una vez aplicado el tratamiento y de la misma forma que al inicio, se realizaron las mediciones de fuerza y porcentaje de grasa 1 semana después de finalizado el programa "postest 1" y 2 semanas después de finalizado el programa "postest 2". El programa tuvo una duración de 12 semanas, a las seis semanas de iniciado el programa se realizó un ajuste de cargas para cada uno de los cuatro ejercicios del programa, con el fin de mantener las intensidades constantes durante el tratamiento.

La asignación aleatoria de las participantes a los grupos de tratamiento se realizó una vez aplicada la técnica por pareo en la variable porcentaje de grasa, ya que fue la que presentó mayor variabilidad.

Los tratamientos asignados al azar fueron los siguientes:

1. Realizar el programa de 3 series de 17 repeticiones al 50\% de 1RM estimada, para un volumen total por ejercicio 2550 unidades $(3 \times 17 \times 50=2550)$.

2. Realizar el programa de 4 series de 8 repeticiones al $80 \%$ de $1 \mathrm{RM}$ estimada, para un volumen total por ejercicio 2560 unidades $(4 \times 8 \times 80=2560)$.

\section{Análisis Estadístico}

Se utilizó un ANOVA 2 x 4 (2 grupos $\mathrm{x} 4$ mediciones) de medidas repetidas para cada una de las variables dependientes. En los casos necesarios, donde la $\mathrm{F}$ dió significativa, se realizó análisis post-hoc. 


\section{RESULTADOS}

La estadística descriptiva (promedio y desviación estándar) para cada grupo en las cuatro mediciones se presenta en la tabla1. En la tabla 2, se muestran los resultados del ANOVA de dos vías para cada una de las pruebas de fuerza, correspondientes a los ejercicios del programa y el $\%$ de grasa corporal.

Tabla 1

Estadística descriptiva para ambos grupos

\begin{tabular}{|c|c|c|c|c|}
\hline & Pretest 1 & Pretest 2 & Postest 1 & Postest 2 \\
\hline \multicolumn{5}{|c|}{ \% Grasa (mm) } \\
\hline Grupo $50 \%$ & $35.62 \pm 7.33$ & $35.69 \pm 7.12$ & $27.66 \pm 7.56$ & $27.75 \pm 7.77$ \\
\hline Grupo $80 \%$ & $37.36 \pm 5.37$ & $37.51 \pm 5.53$ & $28.82 \pm 4.81$ & $28.96 \pm 5.08$ \\
\hline \multicolumn{5}{|c|}{ Flexión Rodilla (lb) } \\
\hline Grupo 50\% & $49.00 \pm 8.94$ & $52.00 \pm 6.70$ & $70.00 \pm 10.00$ & $70.00 \pm 10.00$ \\
\hline Grupo $80 \%$ & $62.00 \pm 20.49$ & $62.00 \pm 20.49$ & $79.00 \pm 16.36$ & $79.00 \pm 13.63$ \\
\hline \multicolumn{5}{|c|}{ Extensión Rodilla (lb) } \\
\hline Grupo $50 \%$ & $74.00 \pm 8.21$ & $74.00 \pm 8.21$ & $90.00 \pm 9.35$ & $90.00 \pm 9.35$ \\
\hline Grupo $80 \%$ & $90.00 \pm 14.14$ & $90.00 \pm 18.70$ & $28.82 \pm 12.54$ & $28.96 \pm 12.54$ \\
\hline \multicolumn{5}{|c|}{ Press de banca (lb) } \\
\hline Grupo $50 \%$ & $41.00 \pm 6.51$ & $42.00 \pm 6.70$ & $58.00 \pm 5.70$ & $58.00 \pm 5.70$ \\
\hline Grupo $80 \%$ & $50.00 \pm 14.57$ & $50.00 \pm 14.57$ & $67.00 \pm 13.50$ & $67.00 \pm 13.50$ \\
\hline \multicolumn{5}{|c|}{ Jalón de Polea (lb) } \\
\hline Grupo $50 \%$ & $73.00 \pm 6.70$ & $73.00 \pm 6.70$ & $83.00 \pm 7.58$ & $83.00 \pm 7.58$ \\
\hline Grupo $80 \%$ & $81.00 \pm 5.47$ & $81.00 \pm 5.47$ & $102.00 \pm 5.70$ & $102.00 \pm 5.70$ \\
\hline
\end{tabular}

Tabla 2

Resumen de ANOVA ( 2 x 4) para las cuatro pruebas de fuerza y el porcentaje de grasa corporal

\begin{tabular}{lll}
\hline Pruebas & Variable & $\left.\mathbf{F ~} \quad \mathbf{( *}^{*} \mathbf{0 . 0 5}\right)$ \\
\hline \multirow{2}{*}{ Press de banca } & Tratamiento & 1.745 \\
& Mediciones & $86.365^{*}$ \\
& Interacción & 0.058 \\
\hline \multirow{2}{*}{ Flexión de rodilla } & Tratamiento & 1.395 \\
& Mediciones & $34.497^{*}$ \\
& Interacción & 0.277 \\
\hline \multirow{2}{*}{ Extensión de rodilla } & Tratamiento & $5.886^{*}$ \\
& Mediciones & $21.780^{*}$ \\
& Interacción & 0.020 \\
\hline \multirow{2}{*}{ Halón de polea frontal } & Tratamiento & $17.153^{*}$ \\
& Mediciones & $40.894^{*}$ \\
\multirow{2}{*}{$\%$ de grasa } & Interacción & $5.149^{*}$ \\
\hline & Tratamiento & 0.142 \\
& Mediciones & $66.110^{*}$ \\
\hline
\end{tabular}


Con base en el análisis estadístico ANOVA 2 x 4 (grupos $\mathrm{x}$ mediciones) de medidas repetidas para la variables press de banca, flexión de rodilla, extensión de rodilla la $\mathrm{F}$ fue significativa para mediciones (ver tabla 2). El análisis post-hoc aplicado, señaló que existen diferencias significativas entre las mediciones pretest 1 y postest 1 y $2(p<0.05)$, así como entre las mediciones pretest $2 \mathrm{y}$ postest 1 y $2(\mathrm{p}<0.05)$, el comportamiento se presenta tanto en el grupo que se ejercitó al 50 $\%$ de intensidad como el que se ejercitó al 80 $\%$ de intensidad (ver figuras de la 1, 2 y 3 ).

Figura 1

Efecto del entrenamiento con pesas en la prueba Press de banca horizontal

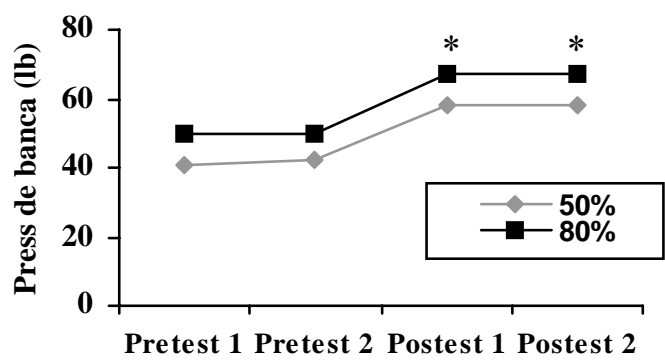

*: significativamente diferente entre Pretest 1 y 2

Figura 2

Efecto del entrenamiento con pesas en la prueba Flexión de rodilla

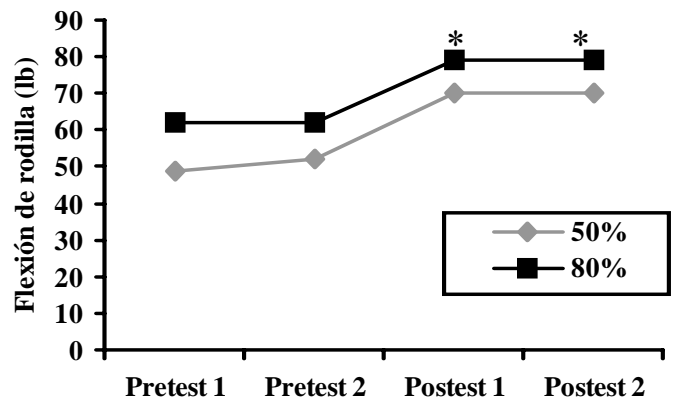

*: significativamente diferente entre Pretest 1 y 2
Figura 3

Efecto del entrenamiento con pesas en la prueba Extensión de rodilla

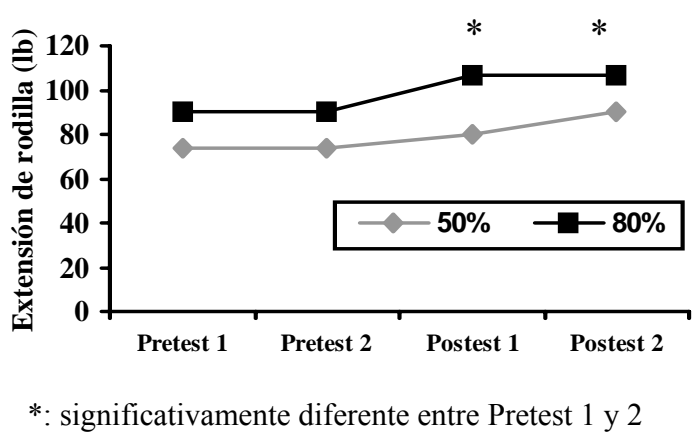

Para la prueba halón de polea como se aprecia en la tabla 2, se obtuvo una $\mathrm{F}$ significativa para la interacción, analizados los efectos simples y post-hoc respectivos se encontró que tanto el grupo que se ejercitó al 50\% como al $80 \%$ de intensidad presentaron diferencias significativas entre el pretest 1 y los postest 1 y $2(\mathrm{p}<0.05)$, así como entre el pretest 2 y los postest 1 y $2(\mathrm{p}<0.05)$, sin embargo el grupo que se ejercitó al $80 \%$ mejoró más que el grupo del $50 \%$ de intensidad (ver figura 5).

Figura 4

Efecto del entrenamiento con pesas en la prueba Halón de Polea al frente

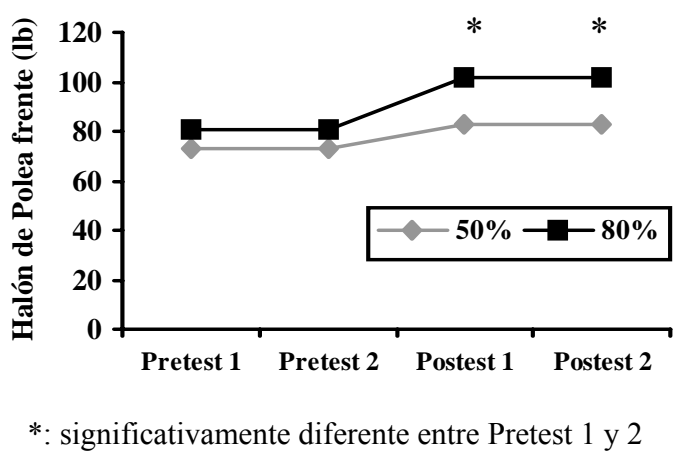

Por último, para el porcentaje de grasa corporal, según el análisis estadístico aplicado ANOVA 2 x 4, hubo diferencia significativa entre mediciones $(\mathrm{p}<0.05) . \quad$ El análisis post-hoc respectivo El análisis post-hoc aplicado, señaló que existen diferencias significativas entre las mediciones pretest 1 y postest 1 y $2(\mathrm{p}<0.05)$, así 
como entre las mediciones pretest 2 y postest 1 y $2(\mathrm{p}<0.05)$, el comportamiento se presenta tanto en el grupo que se ejercitó al $50 \%$ de intensidad como el que se ejercitó al $80 \%$ de intensidad (ver figura 5)

Figura 5

Efecto del entrenamiento con pesas en el porcentaje de grasa corporal

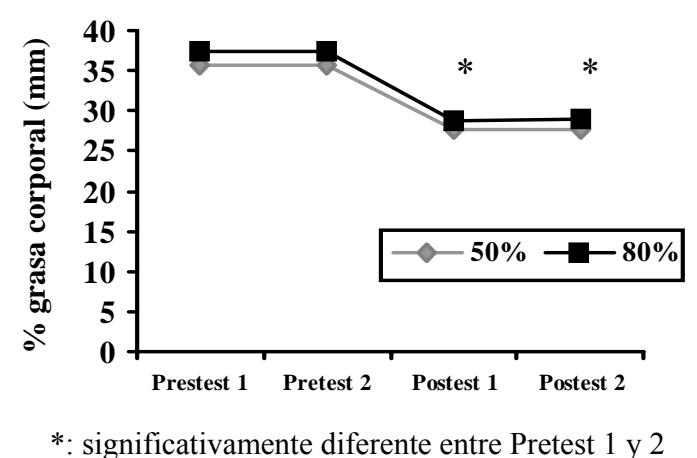

\section{DISCUSIÓN}

La pregunta inicial de este trabajo fue la de observar si se producían cambios en la fuerza y el porcentaje de grasa trabajando a intensidades distintas pero con un volumen de trabajo total semejante.

Los resultados demuestran que hay diferencias estadísticamente significativas entre mediciones, en la ganancia de fuerza y el porcentaje de grasa para ambas intensidades de trabajo, como se ilustra en las figuras 1,2 y 3 . Lo cual demuestra que hay una ganancia de fuerza tanto a intensidades altas $(80 \%)$ como bajas $(50 \%)$ cuando se hacen comparables con el volumen de trabajo total; que es el producto de las series por las repeticiones por el porcentaje de trabajo.

Otros estudios, como el de Kaver y otros (1999) y el de Marx y otros. (2001), también encontraron ganancias de fuerza a a intensidades de ejercicio altas y bajas, controlando el volumen total de trabajo.

Sin embargo, tales resultados no fueron consistentes para la prueba jalón de polea, pues el grupo que se ejercitó al $80 \%$ de intensidad logró una ganancia mayor (ver figura 4). En el jalón de polea frontal, donde el dorsal ancho es, el músculo que ejecuta la acción principal, el $\%$ de cambio fue de $25.9 \%$ para que trabajo al $80 \%$ de intensidad mientras que para el que se ejercitó al $50 \%$ logró un \% de cambio del $13.7 \%$. Estos resultados sugieren que los grupos musculares grandes podrían verse mayormente beneficiados que los grupos musculares pequeños, al ejercitarse a intensidades altas, aun cuando se controle la volumen total de trabajo, sin embargo se requiere de mayor investigación.

Los resultados en relación con la disminución del porcentaje de grasa corporal confirman los encontrados el estudio de Astrup (1999), mostrando una reducción significativa en el $\%$ de grasa corporal al aplicar un programa de entrenamiento con pesas.

En el presente estudio a diferencia de los estudios citados, se controló la variable tiempo. En ningún momento durante la sesión hubo descanso, pues las participantes entre serie y serie del mismo ejercicio, realizaban una serie de otro ejercicio de diferente grupo muscular, así como todos los ejercicios del programa.

Lo que implicó sesiones de 50 minutos ejercicio continuo, lo que sugiere que la reducción en $\%$ de grasa corporal se deba a ello independientemente de la intensidad a la cual se ejercite.

En conclusión, a intensidades del 50\% como del $80 \%$, disminuye el porcentaje de grasa corporal y aumenta la fuerza, para los grupos musculares estudiados, en mujeres con edad comprendida entre los 50 y 59 años, al realizar una misma carga total de ejercicio con pesas. Los hallazgos sugieren que el entrenamiento con pesas a cargas altas, benefician a los grupos musculares grandes.

\section{REFERENCIAS}

Astrand, P. (1992). Physical activity and fitness. American Journal Clinical Nutrition. American Society for Clinical Nutrition. USA, p12315-65.

Astrup, A. (1999). Physical activity and weight gain and fat distribution changes with menopause: current evidence and research issues. Medicine and Science in Sports and Exercise, 31(11), S564-S567.

Bemben, D., Fetters, N., Bemben, N., Koh, E. (2000). Musculoskeletal responses to high-and low-intensity resistance training in early postmenopausal women. 
Medicine and Science in Sportas and Exercise. pp.1949-1957.

Booth, F.W., Barnard, R.J., Blair, S.N. (2001). Skeletal Muscle and Health. Suplemento Medicien and science in Sports and Exercise. 33(5)p.S312

Campos, S., Fonseca, L. y Salazar. W. (1999). Respuestas y adaptaciones de la presión arterial y la frecuencia cardiaca en personas mayores de 60 anos posteriormente a la realización de ejercicios con pesas a diferentes intensidades. En: Boletín informativo de la Escuela de Educación Física y Deportes. San José, Costa Rica: Universidad de Costa Rica.

Campos, C., Fonseca, L. Román, N. (1999) Variación del nivel de lípidos saguíneos en personas activas mayores de 60 años al realizar ejercicio con pesas a diferente intensidad. Revista Gerontológico. CENDEISS-CCSS. (1), 77-87.

Fiatarone, M., Marcks, E., Ryan, N., Meredith, C., Evans, W. (1990). High-intensity strength training in nongenarians: Effect on skeletal muscle. JAMA. 263:3029-3034.

Gutiérrez, R.(1999). La actividad física como medio de rehabilitación de enfermedades crónicas degenerativas. Acta Medica Costarricense, 41, 3743.

Heislein, D.M., Harris, B.A. y Jette, QA.M. (1994). A strength-training program for menopausal women: a pilot study. Archives of Physical Medicine and Rehabilitation, 75(2), 192-204.

Hunter, G., Wetztein, C., Fields, D., Brown, A. y Bamman, D. (2000). Resistance training increases total energy expenditure and free-living physical activity in older adults. Journal of Applied Physiology, 89, 977-984.

Israel, S. (1992). Age-related Changes in Strength and Special Groups. En Komi, P.V. (Ed.). Strength and Power in Sport (pp. 319-328). Oxford, Inglaterra: Blackwell Scientific Publications, Vol. III.

Kaver, R., Fonseca, L., Salazar, W. (1999). El efecto del entrenamiento de un programa de ejercicios con pesas, en la máquina de extensión de rodillas y en el press de banca con cargas del $30 \%$ ó $70 \%$ de 5 RM en el adulto mayor. Revista Gerontológico. CENDEISS-CCSS. (1), 49-62.

Laidlaw, D., Kornatz, K., Keen, D., Suzuki, S. Y Enoka, D.(1999). Strength training improves the steadiness of slow lengthening contractions performed by older adults. Journal of Applied Physiology, 87 (5), 17861795.

Layne, J.E. y Nelson, M. E. (1999). The effects of progressive resistance training on bone density: a review. Medicine and Science in Sports and Exercise, 31 (1), 25 -30.

Marx, j., Ratamess, N., Nindl, B., Gotshlk, L., Volek, J., Dohi, K., Bush, J., Gomez, A. y Kraemer, W. (2001). Low volume circuit versus high volume periodized resistance training in women. Medicine and Science in Sports and Exercise, 33(4), 635-643.

Meredith, C.N., Frontera, W.R., Fischer, E.C. (1989). Peripheral effects of training in young and old Subjects. J. Applied Physiology. 66:2844-2849

Morel, V. (1996). Relaciones entre funcionabilidad, el nivel de actividad físic, cognitivo y anímico de adultos mayores participando en programas recreativos de actividad física. Memoria III Simposio Internacional en Ciencias del Deporte y la Salud. Universidad de Costa Rica, p.8699

Nichols, J.F., Nelson, K.P., Peterson, K.K. y Sartoris, D.J. (1995). Bone mineral density responses to high intensity strength training in active older women. Journal of aging and physical activity, 3 (1), 26-38.

Meléndez, O.A. (2000). Actividades Físicas para mayores: las razones para hacer ejercicio. Editorial GYMNOS, Madrid, España.

Peterson, S. (1991). Muscular strength and bone density weight training in middle- aged women. Medicine and Science in Sports and Exercise, 23 (4), 499-504.

Russo, F.A., Chodzko-Zajko, W.J., Sinning, W.E. (2001). Comparative effects of high-low resistance traning protocols on Muscle functionand functional fitness in older men. Suplemento Medicien and science in Sports and Exercise. 33(5)p.S324.

Smith, G.L.; Odwyer, K.O. Shen-Yu. L y Blanpied, P.R.(1991). The effect of trunk resistive exercise on muscle strength in postmenopausal women.

Journal of Orthopedic and Sports Physical Therapy, 13 (6), 300-309.

Westcott, W. (1991). Fitness management www.exrx.net/fatloss/wt\%26end.html.

Willmore J. y Costill D. (1999). Changes in Strength with Aging. Physiology of Sports and Exercise. 2nda edición. Human Kinetics. U.S.A.. 